\title{
A Cognitive Radio Scheme For Dynamic Resource Allocation Based ON QOE
}

\author{
Jamal Raiyn \\ Computer Science Department \\ Al-Qasemi Academic College, Baka Al Gharbiah, Israel
}

\begin{abstract}
This paper introduces a cognitive radio scheme based on quality of experience (QoE). QoE involves the mobile end user's preferences. Considering QoE can lead to an improved cognitive radio resource management strategy. The cognitive radio scheme aims to manage the traffic flow in dynamic systems; it is a new way of thinking about dynamic resource management in cellular systems. The Use of the cognitive radio techniques in cellular systems will improve the resource assignment in wireless communication. The cognitive radio is a self-aware communication system that aims to use the resource assignment in an efficient way. The proposed algorithm is very successful at handling the call-blocking rate based on QoS and $Q o E$.
\end{abstract}

\section{KEYWORDS}

cognitive radio, cellular system, call-blocking rate, QoE

\section{INTRODUCTION}

To increase resource allocation efficacy in cellular systems, various resource allocation schemes have been proposed (Shai et al., 2011; Xin et al., 2001). The goal of resource allocation strategies is to decrease new call-blocking probabilities and the handoff call-blocking rate (Raiyn, 2009, 2012). The call-blocking rate is determined by urban traffic congestion. Over the last two decades, the demand for developing a real-time traffic prediction methodology that is robust and accurate enough to handle the urban traffic has grown rapidly. One of the biggest challenges is the forecasting of abnormal conditions in dynamic traffic management (Chrobok et al., 2000; Zhang et al., 2003). Another is to fulfill the mobile users' demands and satisfy their preferences under the constraint of real-time predictions. This is a challenge because resource demand in the system is regularly exceeded. There are three major strategies for relieving congestion (Raiyn, 2010 , ); the first strategy is to increase the number of base stations; however, this is very expensive and can only be accomplished in the long term. The second strategy is to limit traffic demand or to make data communications more expensive, which will be strongly disapproved of by mobile users. The third strategy focuses on the efficient and optimized utilization of existing resources. This strategy is the best trade-off and is garnering more and more attention. Currently, the cognitive radio technology is the most promising approach to implementing the third strategy. The term cognitive radio (CR) is defined according to (Akyildiz et al., 2008) as an adaptive, intelligent radio and network technology that can automatically detect available resources in a cellular networks and change transmission parameters, to improve radio-operating behavior. In order to decrease the call-blocking rate, we introduced a cognitive radio scheme for resource allocation based on quality of experience $(\mathrm{QoE})$. The term $\mathrm{QoE}$ is defined and relates to how end DOI : 10.5121/ijwmn.2016.8101 
users perceive the quality of an application or service (Tang et al., 2014). The cognitive radio system aims to allow universal maximization of spectrum utilization via environmental sensing (Han et al., 2011; Kim, et al., 2001). Cognitive radio changes its transmitter parameters by interacting with the environment it operates in, and it requires a highly reliable sensing technique. Cooperative sensing technologies have been studied to meet this need. To manage incomplete information on environmental sensing (ES), we have introduced a forecasting scheme (Wild, 1997).

The introduction of a forecasting scheme based on QoE will lead to adaptive cognitive radio technology based on the computation and evaluation of QoE. Adaptive cognitive radio technology considers shadow fading in the coverage zone. Many studies have shown that urban traffic congestion increases the new call-blocking rate. Over the last two decades, the demand for developing a traffic prediction methodology that is robust and accurate enough to handle dynamic resource allocation has grown rapidly. One of the biggest challenges in dynamic resource allocation is predicting abnormal conditions. Introducing a prediction scheme will allow the cognitive radio to universally maximize spectrum utilization by environmental sensing. This paper is organized as follows. In the second section we introduce the system model. In the third section, we analyze and evaluate the performance of several forecasting schemes.

\section{2 .THE SYSTEM MODEL}

A cellular network (Zysman \& Menkes, 2001) consists of an array of cells. We partitioned the cellular network into clusters. For each cluster, which consists of a number of cells, we set a control system, an agent, which manages the cell. When a mobile host in a cell needs to communicate, it sends a request to the centralized distributor to acquire a channel (Raiyn, 2011b). The centralized distributor then attempts to identify a vacant channel. Hence, the centralized distributor informs the mobile host about which channel it can use. The system focuses on handoff (Raiyn, 2009, 2011a) events, and control of a phone in current use is handed over from one cell to another. The handover starts when the received power is weak and falls below a given target value, $\mathrm{SNR}^{\mathrm{tgt}}$. The handover is performed when the mobile station (MS) receives a weak SNR from BS- $A$ and, at the same time, a strong signal from the neighbour BS, i.e.,

$$
S N R_{A}^{t g t}>P_{r}>S N R_{B}^{t g t}
$$

where $P_{r}$ is the received power signal at the mobile hot $(\mathrm{MH})$ location; in other words, the handoff strategy is designed as follows: At first, we track changes in the SNR of the current channel. When the SNR is above threshold SNR ${ }^{\text {tgt }}$, a handoff will not occur. If the SNR is below threshold SNR ${ }^{\text {tgt }}$, we will begin handoff execution immediately. In another case, when the SNR falls between the two thresholds, we will use our handoff initiation algorithm to make a decision. The adaptive agent decides whether the mobile host (MH) should hand off or not, based on a combination of several parameters, e.g., the SNR and the system load, in order to avoid repeating or performing unnecessary handoffs (Raiyn, 2012b). The system load is used to decrease the handoff-dropping rate and to provide a QoS guarantee.

\subsection{CR based on QoE}

Many approaches based on quality of service (QoS) have been proposed to maximize overall system performance, such as throughput, delay and jitter (Riyn, 2012b). However, the requirements of different applications differ in terms of network resources. Moreover, QoS 
International Journal of Wireless \& Mobile Networks (IJWMN) Vol. 8, No. 1, February 2016

cannot directly reflect the perceived quality of services by end users, which may cause a waste of radio resources. To cope with the rising traffic volume and different requirements of emerging applications, efficient resource allocation technologies are much needed for cellular systems. Currently, since QoE connects as closely as possible with users' subjective perceptions, it plays a more important role in indicating users' experiences and reactions to certain services or applications. Thus, network operators have broadened their focus from network QoS to user QoE. QoE, which assesses the performance of a network from the user's viewpoint, is drawing more and more attention. Dynamic resource allocation schemes for QoE optimization have not yet been studied, yet, according our primary results, these could produce improvements in users' perceived quality (Raiyn, 2007). In some cases, it is difficult, sometimes even impossible, to obtain complete information from the QoE model in advance. In contrast to traditional QoS, which attempts only technical measurements of multimedia transmissions, QoE is basically a subjective measurement of end-to-end multimedia service from the user's point of view. Where information from the QoE model may be incomplete, especially under abnormal conditions, we have introduced forecasting schemes. The next section will discuss these. Cognitive radio management considers QoE by forecasting dynamic traffic patterns under abnormal conditions (Toledo, 2014).

\subsection{Coverage planning}

In this section, we give an overview of spread spectrum and the propagation of radio signals. The propagation of radio signals in both uplinks and downlinks is affected by the physical channel in several ways:

- A signal propagating, due various reasons, usually arrives at its destination along a number of different paths, referred to as multi-paths. These paths arise from scattering, reflection, refraction or diffraction of the radiated energy of objects in the environment, as shown in Figures 1 and Figure 2. The received signal is much weaker than the transmitted signal, due to phenomena such as mean propagation loss, slow fading and fast fading.

- Mean propagation loss is caused by square-law spreading, absorption by water and the effects of ground reflections such high building. It is range-dependent and changes very slowly, even for fast mobiles. Slow fading results from blockage by buildings and natural features and is also known as long-term fading, or shadowing. Fast fading results from multi-path scattering in the vicinity of the mobile. It is also known as short-term fading or Rayleigh fading, for reasons explained below. Multipath propagation results in the spreading of the signal in different dimensions. Figure 3 illustrates the resource demand at the base station.

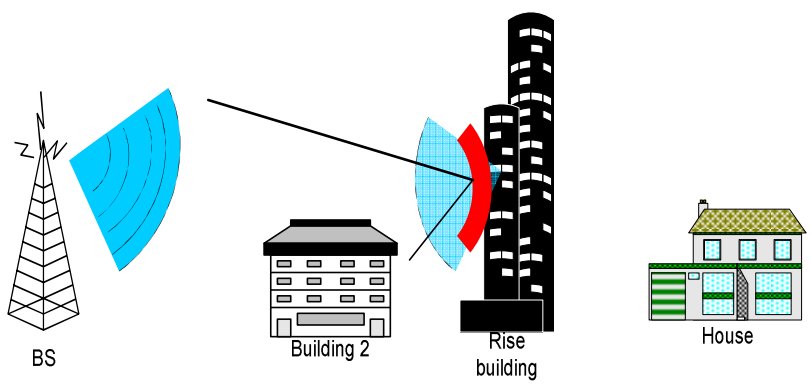

Figure 1: Signal reflection. 


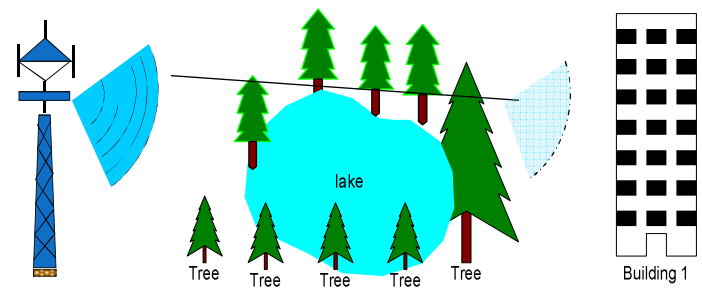

Figure 2: Signal absorption.

\subsection{Coverage map}

Developing new simulator-based cognitive radio is necessary. Simulator-based cognitive radio considers real-time resource allocation, environmental change and base station mobility. Furthermore, the simulator takes into account trees and high-rise buildings that affect resource allocation. This section illustrates the process of radio coverage prediction (Shi et al., 2011) by introducing a case study that was undertaken for radio network planning (Xin et al., 2001) using coverage planning, as illustrated Figure 3. This study in an urban environment concluded that traditional propagation model could lead to increase the call blocking rate, with less-thanexpected quality of service, unacceptable interference, and more base stations than necessary. Using the coverage-planning platform, the radio network designer can reach optimal levels for base station deployment and configuration, while meeting expected service level requirements. This can be done with the help of propagation models, geographical information, filed data and optimization algorithms that are integrated into the planning tool. In order to meet the requirements of the mobile services, the radio network must offer sufficient coverage and capacity, while maintaining the lowest possible deployment costs. As mentioned in the previous section, the network in a cellular network is divided into many cells, and usually a base station is deployed in the center of each cell (Raiyn, 2008). For the sake of easy analysis, the cells are represented as neighbouring hexagons, while in reality they can be any form and can overlap each other. The size of each cell, when fixed, will usually stay stable. Unlike the CSMA system described above, there is one important feature in GSM network planning: coverage planning and capacity planning are independent. The coverage planning depends on the received signal strength; that is, the covered area is limited almost only by the minimum signal strength at the cell range, while the capacity planning depends mainly on frequency allocation.

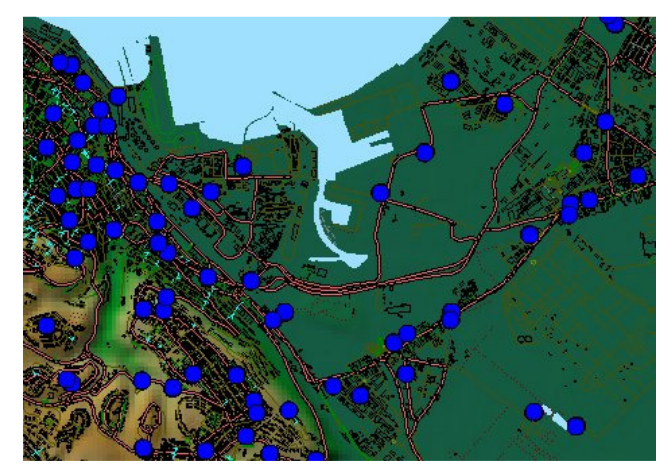

Figure 3: Base station sites. 
Figure 4 shows a three-sector antenna pattern, which is clear from the shape of the results. Figure 5 shows the deployment in the suburbs of Portland and the coverage sectors; the colors represent the frequency channel used for each sector. This plan is for a WiMAX system - a system considered to be the precursor of the fourth generation of cellular networks. In a WiMAX system, orthogonal planning, like GSM, and non-orthogonal planning, like that of CDMA networks, can be combined. Hence, we see that the same channel is allocated to neighboring sectors, and the interference between them should then be mitigated by other means.

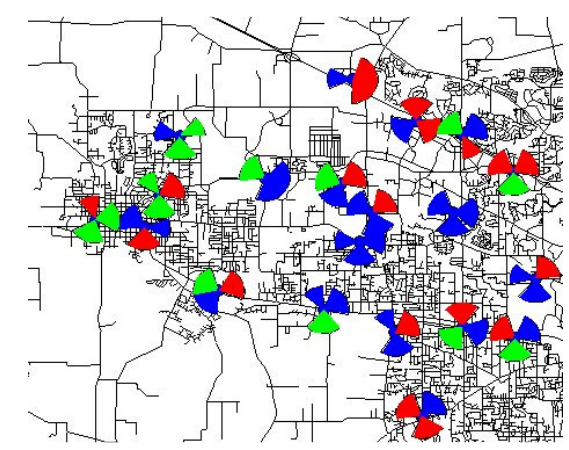

Figure 4: Base station deployment and frequency plan.

Figure 5 shows the results of base station transmission and the impact of streets and buildings on propagation. The different colors in the image represent the coverage areas of three base stations (shown as blue dots with a number next to them). Yellow represents the area where the signal arriving from station 1 is strongest, green represents the signal from station 4 , and red the signal from station 5. Stations 2 and 3 are not shown in the picture; nevertheless, there are some points where their signal is strongest and can be seen colored in pink or blue (Raiyn, 2015a/b).

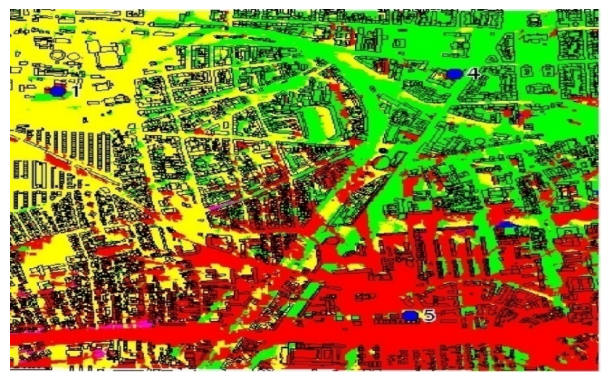

Figure 5: Signal strength.

\subsection{Resource allocation-based QoE}

Coverage planning and coverage maps give a picture of resource allocation for static traffic loads. To manage dynamic resource allocation-based QoE, we introduced a cognitive radio scheme.

A cognitive radio allocates the dynamic resources in a cellular system based on environmental sensing (Raiyn, 2008). Cognitive radio can change its transmitter parameters, which makes it desirable when interact with the environment in which it operates call for a highly reliable 
International Journal of Wireless \& Mobile Networks (IJWMN) Vol. 8, No. 1, February 2016

sensing technique. Spectrum sensing is defined in (Raiyn, 2011b) as the task of finding spectrum holes by sensing the radio spectrum. The term spectrum holes (Raiyn, 2009) refers to sub-bands of the radio spectrum that are underutilized (in part or in full) at a particular instant of time and in a specific geographic location. To be specific, the task of spectrum sensing in this paper involved the detection of spectrum holes. The detection algorithm was based on an exponential moving average. Environmental sensing is needed for cognitive radio to update its local information. Hence, the cognitive radio reassigns dynamic resources to decrease the call-blocking rate. Figure $6(a-c)$ illustrates the demand for resources in a heavy traffic load during peak hours.

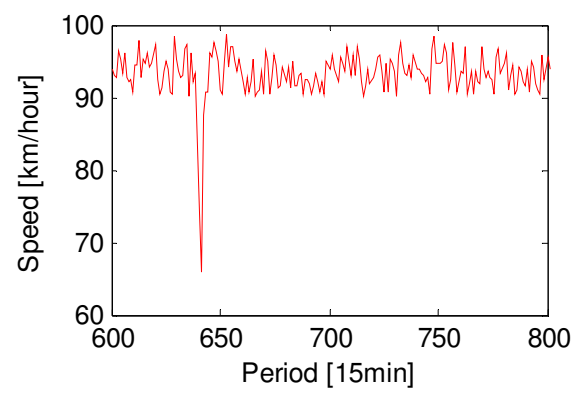

Figure 6a: Resource demands of users.

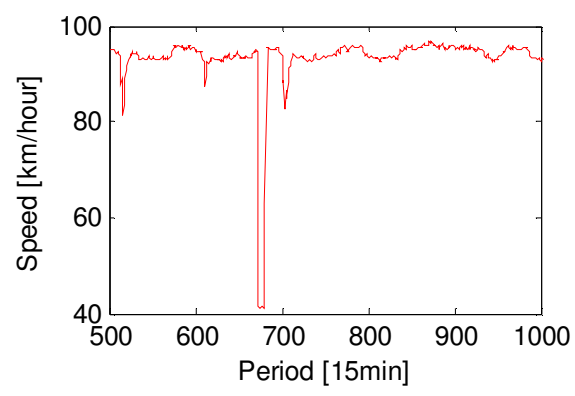

Figure 6b: Resource demands of users.

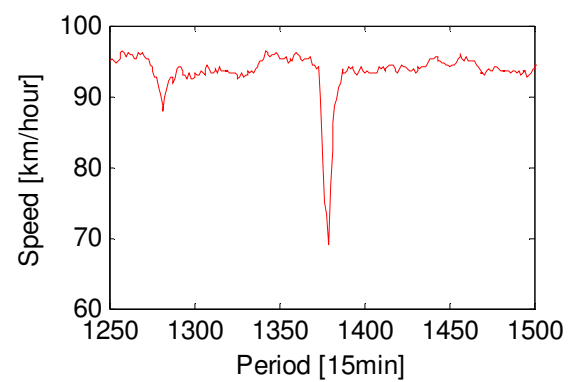

Figure 6c: Resource demands of users. 
International Journal of Wireless \& Mobile Networks (IJWMN) Vol. 8, No. 1, February 2016

\subsection{Evaluation of forecasting performance}

A short-term forecasting scheme was used to manage dynamic resources under abnormal conditions (Toledo at. al.2012, 2014). This section evaluates and analyses forecasting schemebased traffic data. The information was collected via mobile phone service. Based on the WEKA platform, we analysed and compared different prediction schemes, as illustrated in Figure 7. The WEKA (Waikato Environment for Knowledge Analysis) is a platform that includes machinelearning algorithms for data-mining services. It contains tools for data pre-processing, classification, regression, clustering, association rules and visualization. We have used the WEKA to make comparisons between the following schemes:

i. $\quad$ smoothed linear models (LM)

ii. tree decisions (TD)

iii. the nearest-neighbor classifier scheme (NN).

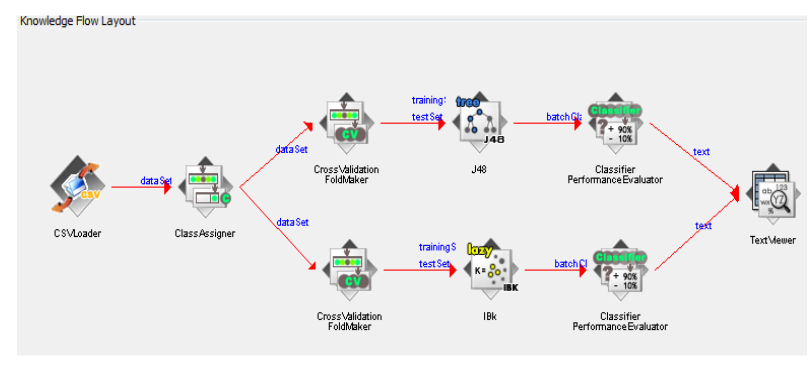

Figure 7: Knowledge flowchart.

In addition, we used Matlab to evaluate forecasting scheme performance. The comparison focused on various statistical measurements of error: mean absolute error (MAE), root mean squared error (RMSE), relative absolute error (RAE) and root relative squared error (RRSE) (Toledo, 2012). Table 1 shows a general comparison between information collected via cellular mobile service and information received by sensors. The results of the quality measurements are summarized in Tables 1-3. Table 3 illustrates that the Nearest Neighbor Scheme offers the clearest and best results compared to the linear model and tree decision schemes.

Table 1: Cellular Versus Sensor Data Based on the LM

\begin{tabular}{|l|l|l|}
\hline \multirow{2}{*}{$\begin{array}{l}\text { Statistical } \\
\text { Measur. }\end{array}$} & \multicolumn{2}{|l|}{ Linear model-LM } \\
\cline { 2 - 3 } MAE & Cellular & Sensor \\
\hline RMSE & 7.973 & 7.1967 \\
\hline RAE & 11.6976 & 11.5308 \\
\hline RRSE & $41.4674 \%$ & $69.6342 \%$ \\
\hline
\end{tabular}


International Journal of Wireless \& Mobile Networks (IJWMN) Vol. 8, No. 1, February 2016

Table 2: Cellular Versus Sensor Data Based on TD

\begin{tabular}{|l|l|l|}
\hline \multirow{2}{*}{$\begin{array}{l}\text { Statistical } \\
\text { Measur. }\end{array}$} & \multicolumn{2}{|l|}{ Tree decision-TD } \\
\cline { 2 - 3 } & Cellular & Sensor \\
\hline MAE & 9.5974 & 7.57 \\
\hline RMSE & 14.0847 & 11.6718 \\
\hline RAE & $49.916 \%$ & $73.2468 \%$ \\
\hline RRSE & $59.1237 \%$ & $69.753 \%$ \\
\hline
\end{tabular}

Table 3: Cellular Versus Sensor Data Based on NN

\begin{tabular}{|l|l|l|}
\hline \multirow{2}{*}{$\begin{array}{l}\text { Statistical } \\
\text { Measur. }\end{array}$} & \multicolumn{2}{|l|}{ Nearest Neighbour } \\
\cline { 2 - 3 } MAE & Cellular & Sensor \\
\hline RMSE & 6.4734 & 6.6224 \\
\hline RAE & 10.1594 & 11.0445 \\
\hline RRSE & $33.6678 \%$ & $64.0777 \%$ \\
\hline
\end{tabular}

A. Traffic flow management

Various travel flow management strategies have been introduced to reduce traffic congestion. The moving average scheme offers best results compared to other forecasting schemes. In this section, we introduce the smoothed moving average scheme for managing traffic flow. However, we distinguish between three main kinds of moving average: the simple moving average (SMA) (Toledo, 2012), the weighted moving average (WMA) (Toledo, 2014) and the exponential moving average (EMA) (Toledo, 2014). A simple moving average is the unweighted mean of the previous data points in the time series. A weighted moving average is a weighted mean of the previous data points in the time series; this average is more responsive to recent movements than a simple moving average. An exponentially weighted moving average (EMA) is an exponentially weighted mean of previous data points; it can be expressed as a proportional percentage:

$$
t t^{F}(t)=\alpha * t t(t)+(1-\alpha) * t t(t-1)
$$

where $t t^{F}(t)$ is the travel time forecast at time $t, \propto$ is the smoothing parameter, and $0 \leq \propto \leq 1$. Alpha determines how responsive a forecast is to sudden jumps and drops. It is the percentages weight given to the prior period, and the remainder is distributed to other historic periods (Raiyn, 2011b). The advantage of all exponential smoothing methods lies in their assigning less weight to past cases than recent ones.

The EMA forecasting model offers impressive forecasting performance compared to actual observations, as illustrated in Figure 8. The moving average scheme yields good results. Table 4 compares the weight factors for an exponentially smoothed, four-month moving average, using a simple moving average that weights every week equally, and a weighted moving average. 
International Journal of Wireless \& Mobile Networks (IJWMN) Vol. 8, No. 1, February 2016

Table 4: SMA, WMA and EMA Compared

\begin{tabular}{|l|l|l|l|}
\hline $\begin{array}{l}\text { statistical } \\
\text { Measurements }\end{array}$ & SMA & WMA & EMA \\
\hline MAE & 6.22 & 8.11 & 5.17 \\
\hline RMSE & 12.33 & 14.04 & 9.57 \\
\hline RAE & 11.84 & 16.54 & 11.54 \\
\hline $\begin{array}{l}\text { Theil's } \\
\text { Coefficient }\end{array}$ & 7.21 & 9.55 & 5.61 \\
\hline
\end{tabular}

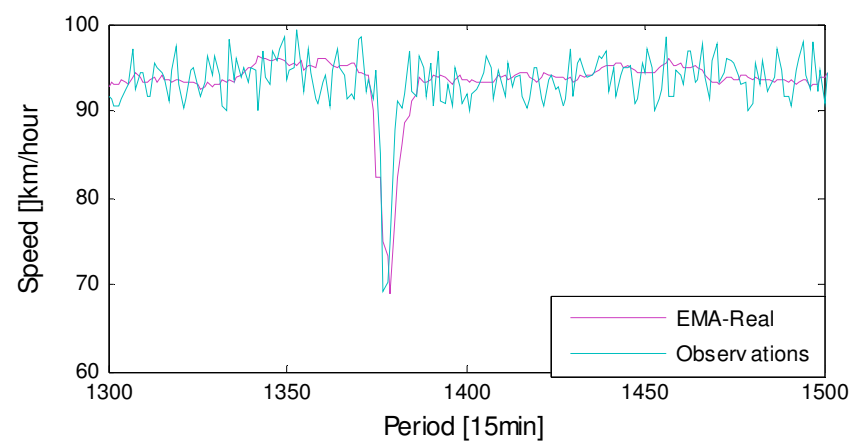

Figure 8: Real-time Forecasting.

\section{CONCLUSION}

Various prediction schemes have been proposed to manage traffic flow. In order to select the best prediction strategy, we analysed and compared different forecasting schemes. In this paper, we have introduced various forecasting schemes based on historical data. Furthermore, we have discussed and summarized some prediction methods based on an analysis of their performance. We conclude that using the exponential moving average is the most accurate method when few data are available. To handle the growth of massively multi-users of UE's, a self-optimized, cognitive radio resource allocation scheme based on environment sensing is needed.

\section{REFERENCES}

[1] Akyildiz, I.F., Won-Yeol, L., Vuran, M.C. and Mohanty, S. (2008). A Survey on Spectrum Management in CognitiveRadio Networks ,IEEE Communications Magazin,. Vol. 46, Issue 4, 40-48.

[2] Chrobok, R., Kaumann, O., Wahle, J. and Scheckenberg, M. (2000). Three Categories of Traffic Data: Historical, Current, and Predictive”, 9th IFAC Symposium, Braunschweig, Germany, 13-15, Vol. 1, June. 221-226.

[3] Han, W., Li, J., Tian, Z. and Zhang, Y. (2011).Dynamic Sensing Strategies for Efficient Spectrum Utilization in Cognitive Radio Networks", IEEE Transactions on Wireless Communications, Vol. 10, No. 11, 3644-3655.

[4] Kim, J-S. Li, G. and Giannakis, B.G.(2001). Multi-Band Cognitive Radio Spectrum Sensing for Quality-of-Service Traffic, IEEE Transactions on Wireless Communications, Vol. 10, No. 10, October. 3506-3515. 
International Journal of Wireless \& Mobile Networks (IJWMN) Vol. 8, No. 1, February 2016

[5] Raiyn, J. (2007). Development and Comparison between Resource Allocation Strategies based on Intelligent Scheme and Traditional Distributed Dynamic Resource Allocation Strategies in Mobile Communication Networks, Wireless Personal Communications, 40, 4, 495-509.

[6] Raiyn, J. (2008). Toward Cognitive Radio Handover Management Based on Social Agent Technology for Spectrum Efficiency Performance Improvement of Cellular Systems, IEEE 19th International Symposium on Personal, Indoor and Mobile Radio Communications (PIMRC- 2008), 15-18 September, Cannes, France.

[7] Raiyn, J. (2009). Developing Cognitive Radio Approach Based on Dynamic SNR to reduce Handoff Latency in Cellular Systems, International Symposium on Performance Evaluation of Computer and Telecommunication Systems, July 13-16, Istanbul, Turkey, 231-237.

[8] Raiyn, J. (2010). Spectrum Efficiency Improvement Based on the Cognitive Radio Management, International Journal of Communications, Network and System Sciences, 3, 3, 280-288.

[9] Raiyn, J. (2011a). Social Agent for Dynamic Resource Management in Heavy Traffic Load Conditions, International Journal on Communications Antenna and Propagation. 1, 5, 418-422.

[10] Raiyn, J. (2011b). A Self-Optimization of the Dynamic Resource Management Based on the Cognitive Radio, Wireless Engineering and Technology, 2, 87-92.

[11] Raiyn, J. (2012a). Handoff Self-Management Based on SNR in Mobile Communication Networks. International Journal of Wireless and Mobile Computing. 6, 1, 39-48.

[12] Raiyn, J. (2012b). Power Control Based on Cognitive Radio Managements: From Theory to Practice, International Journal on Communications Antenna and Propagation, 2, 5, 276-282.

[13] Raiyn, J. (2015a). Using Cognitive Radio Scheme for Big Data Traffic Management in Cellular Systems, International Journal of Information Technology and Management, 14, 2/3.

[14] Raiyn, J. (2015b). Modern Information and Communication Technology and their Application in CyberParks, Journal of Multidisciplinary Engineering Science and Technology, 2, 8, 2178-2183.

[15] Shi, Y. and Hou, T. (2011). Maximizing Capacity in Multihop Cognitive Radio Networks under the SINR Model, IEEE Transactions on Mobile Computing, Vol. 10, No.7. 954-967.

[16] Tang, P., Wang, P., Wang, N. and Nguyen, V.N. (2014). QoE-Based Resource Allocation Algorithm for Multi-Applications in Downlink LTE Systems (2014). International Conference on Computer, Communications and Information Technology. Shanghai, China, 4-7.

[17] Toledo, T. and Raiyn, J. (2012). Performance Analysis and Evaluation of Short-Term Travel Forecast Schemes Based on Cellular Mobile Services. International Review of Civil Engineering. 3, 2, 201205.

[18] Toledo, T. and Raiyn, J. (2014). Real-Time Short-Term Forecasting Based on Information Management, Journal of Transportation Technologies, 4, 11-21.

[19] Wild, D. (1997). Short-term forecasting based on a transformation and classification of traffic volume time series, International Journal of Forecasting 13. pp. 63-72.

[20] Zhang, X., Rice, J.A. Short-term travel time prediction, Transport. Res. Part C: Emer. Technol. 11 (34), 2003.187-210.

[21] Zysman, G.I. and Menkes, H. (2001). Wireless Mobile Communications at the Start of the 21st Century", IEEE Communications Magazine, 2001.

[22] Xin, C., Song, M. MA, L. and Shen, C_C. (2001). Performance Analysis of a Control-Free Dynamic Spectrum Access Scheme, IEEE Transactions on Wireless Communications, Vol. 10, No. 12, December. 4316-4323. 